\title{
DNA Methylation Landscape of 16 Canine Somatic Tissues by Methylation- Sensitive Restriction Enzyme-Based Next Generation Sequencing
}

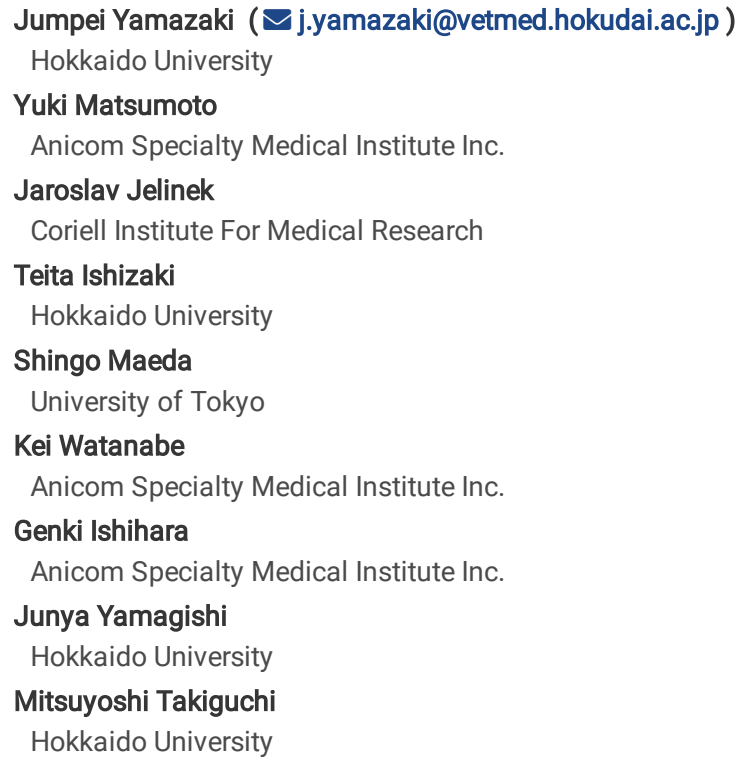




\section{Abstract}

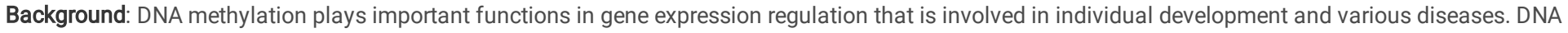
methylation has been well studied in human and model organisms, but only limited data exist in companion animals like dog.

Results: Using methylation-sensitive restriction enzyme-based next generation sequencing (Canine DREAM), we obtained canine DNA methylation maps from 16 somatic tissues. In total, we evaluated $130,861 \mathrm{CpG}$ sites. The majority of $\mathrm{CpG}$ sites were either highly methylated (>70\%, 52.5\%-64.6\% of all CpG sites analyzed) or unmethylated $(<30 \%, 22.5 \%-28.0 \%$ of all $\mathrm{CpG}$ sites analyzed) which are methylation patterns similar to other species. The overall methylation status of $\mathrm{CpG}$ sites across the 32 methylomes were remarkably similar. However, the tissue types were clearly defined by principle component analysis and hierarchical clustering analysis with DNA methylome. We found $6416 \mathrm{CpG}$ sites located closely at promoter region of genes and inverse correlation between DNA methylation and gene expression of these genes.

Conclusions: Our study provides basic dataset for DNA methylation profiles in dogs.

\section{Introduction}

DNA methylation is the conversion of cytosine to 5-methylcytosine at cytosine-guanine $(\mathrm{CpG})$ dinucleotides, causing structural change in the interactions between DNA and protein(s). This reaction is catalyzed by DNA methyltransferases (DNMTs) in mammalian cells and DNA methylation occurring at promoter regions of genes represses gene transcription ${ }^{1}$. Clusters of $\mathrm{CpG}$ sites called $\mathrm{CpG}$ islands (CGI) are found in approximately half of gene promoters in mammalian species ${ }^{2}$. In addition, global methylation at $\mathrm{CpG}$ loci throughout the genome is thought to reflect change in response to the environment, exposure, immune response, and the carcinogenic process. ${ }^{3}$ DNA methylation is a widely recognized regulatory mechanism that is indispensable for cellular development, tissue differentiation, inactive $\mathrm{X}$ chromosome in women ${ }^{4}$, and in imprinting ${ }^{5,6}$.

Recently, genome-wide analyses of DNA methylation have revealed that a number of genes are unequivocally differentially methylated in a variety of normal cells as well as tumors ${ }^{7,8}$. However, genome-wide DNA methylation patterns have been well studied only in humans and rodents ${ }^{9-11}$. The sequencing and analysis of the dog genome ${ }^{12}$ will greatly accelerate the dog as a biomedical and spontaneous model for many diseases in humans such as tumors ${ }^{13}$. Although global hypomethylation or hypermethylation at single loci were found in dogs ${ }^{14-17}$, these studies did not search for changes in genomic location modified by DNA methylation. Given the fact that understanding of DNA methylation pattern in dogs is limited compared to humans and rodents, we previously established Canine DREAM, which is genome-wide DNA methylation analysis in dogs based on next-generation sequencing of methylation-specific signatures created by sequential digestion with Smal and Xmal restriction enzymes ${ }^{18}$. In this study, we aimed to construct genome-wide DNA methylation status in 16 normal cell/tissues in dogs for comprehensive understanding of DNA methylation. This study provides basic information on the dog methylome and a reference data for further study of DNA methylation and comparative animal research.

\section{Results}

\section{CpG sites analyzed}

We studied the DNA methylomes of 32 normal dog tissue samples (16 tissue types) including, lung, heart, stomach, duodenum, ileum, colon, liver, pancreas, adrenal gland, kidney, spleen, muscle, skin (white-colored), oral mucosa (white-colored and black-colored), and bone marrow. A complete list of cell and tissue types are shown in Table 1. 
Table 1

Tissue types used in this study.

\begin{tabular}{|c|c|c|c|c|c|c|c|}
\hline & Samples & $\begin{array}{l}\text { Number of } \\
\text { reads }\end{array}$ & $\begin{array}{l}\text { Number of CpG } \\
\text { sites covered }\end{array}$ & $\begin{array}{l}\text { Average CpG methylation } \\
\text { per sample }\end{array}$ & $\begin{array}{l}\text { Percent } \\
\text { methylated } \\
(>=0.7)\end{array}$ & $\begin{array}{l}\text { Percent } \\
\text { unmethylated }(<= \\
0.3 \text { ) }\end{array}$ & $\begin{array}{l}\text { Percent intermediately } \\
\text { methylated } \\
(>0.3 \text { and }<0.7)\end{array}$ \\
\hline 1 & Lung 73 wk & $45,353,568$ & 181,096 & 61.5 & 56.5 & 24.8 & 18.7 \\
\hline 2 & Lung 58 wk & $35,466,811$ & 169,334 & 61.7 & 56.7 & 24.6 & 18.7 \\
\hline 3 & Heart 73 wk & $34,954,413$ & 170,173 & 61.5 & 56.8 & 24.6 & 18.6 \\
\hline 4 & Heart 58 wk & $31,019,481$ & 166,354 & 61.1 & 55.8 & 24.6 & 19.5 \\
\hline 5 & $\begin{array}{l}\text { Stomach } 73 \\
\text { wk }\end{array}$ & $38,545,997$ & 174,204 & 64.1 & 61.1 & 24.0 & 14.9 \\
\hline 6 & $\begin{array}{l}\text { Stomach } 58 \\
\text { wk }\end{array}$ & $37,724,714$ & 173,994 & 61.9 & 57.5 & 25.3 & 17.2 \\
\hline 7 & $\begin{array}{l}\text { Duodenum } \\
73 \text { wk }\end{array}$ & $41,399,337$ & 175,666 & 61.3 & 55.7 & 23.8 & 20.5 \\
\hline 8 & $\begin{array}{l}\text { Duodenum } \\
58 \mathrm{wk}\end{array}$ & $47,420,647$ & 176,964 & 61.2 & 57.1 & 26.6 & 16.3 \\
\hline 9 & Ileum 73 wk & $24,531,063$ & 156,739 & 63.0 & 58.3 & 23.2 & 18.5 \\
\hline 10 & Ileum 58 wk & $50,538,113$ & 178,711 & 62.8 & 58.9 & 24.8 & 16.3 \\
\hline 11 & Colon 73 wk & $37,269,930$ & 166,719 & 62.2 & 57.0 & 23.7 & 19.3 \\
\hline 12 & Colon 58 wk & $37,292,174$ & 171,064 & 60.9 & 54.5 & 24.1 & 21.4 \\
\hline 13 & Liver 73 wk & $33,074,566$ & 166,541 & 61.1 & 55.7 & 24.8 & 19.5 \\
\hline 14 & Liver 58 wk & $41,659,584$ & 173,433 & 59.4 & 52.8 & 25.5 & 21.8 \\
\hline 15 & $\begin{array}{l}\text { Pancreas } 73 \\
\text { wk }\end{array}$ & $35,810,465$ & 165,875 & 60.1 & 54.9 & 27.4 & 17.7 \\
\hline 16 & $\begin{array}{l}\text { Pancreas } 58 \\
\text { wk }\end{array}$ & $39,201,439$ & 171,747 & 60.1 & 54.9 & 26.9 & 18.3 \\
\hline 17 & $\begin{array}{l}\text { Adrenal } \\
\text { gland } 73 \text { wk }\end{array}$ & $36,444,210$ & 165,838 & 60.9 & 56.0 & 26.1 & 17.9 \\
\hline 18 & $\begin{array}{l}\text { Adrenal } \\
\text { gland } 58 \text { wk }\end{array}$ & $34,960,686$ & 170,611 & 63.1 & 59.1 & 23.3 & 17.6 \\
\hline 19 & Kidney 73 wk & $33,793,352$ & 170,886 & 61.2 & 56.3 & 26.2 & 17.5 \\
\hline 20 & Kidney 58 wk & $40,913,656$ & 173,920 & 60.8 & 55.6 & 26.5 & 17.9 \\
\hline 21 & Spleen 73 wk & $44,270,510$ & 173,463 & 64.3 & 60.8 & 23.0 & 16.2 \\
\hline 22 & Spleen 58 wk & $31,708,030$ & 163,656 & 65.1 & 62.4 & 22.5 & 15.1 \\
\hline 23 & $\begin{array}{l}\text { Muscle } 73 \\
\text { wk }\end{array}$ & $29,969,294$ & 163,433 & 59.4 & 52.9 & 25.5 & 21.6 \\
\hline 24 & $\begin{array}{l}\text { Muscle } 58 \\
\text { wk }\end{array}$ & $44,497,941$ & 167,724 & 59.0 & 52.5 & 26.1 & 21.4 \\
\hline 25 & $\begin{array}{l}\text { Skin }(W) 73 \\
\text { wk }\end{array}$ & $43,810,934$ & 174,647 & 59.9 & 53.6 & 25.5 & 20.9 \\
\hline 26 & $\begin{array}{l}\text { Skin }(W) 58 \\
w k\end{array}$ & $35,955,601$ & 167,385 & 60.3 & 55.0 & 26.6 & 18.4 \\
\hline 27 & $\begin{array}{l}\text { Oral }(W) 73 \\
\text { wk }\end{array}$ & $34,782,022$ & 164,878 & 60.6 & 56.6 & 28.0 & 15.5 \\
\hline 28 & $\begin{array}{l}\text { Oral (W) } 58 \\
\text { wk }\end{array}$ & $38,799,946$ & 168,123 & 61.5 & 56.6 & 24.9 & 18.6 \\
\hline 29 & $\begin{array}{l}\text { Oral (B) } 73 \\
\text { wk }\end{array}$ & $34,654,969$ & 169,574 & 59.5 & 53.6 & 26.4 & 20.0 \\
\hline 30 & $\begin{array}{l}\text { Oral (B) } 58 \\
\text { wk }\end{array}$ & $48,856,203$ & 178,170 & 60.2 & 54.9 & 26.8 & 18.3 \\
\hline 31 & BM 73 wk & $34,852,223$ & 176,838 & 64.2 & 64.5 & 23.4 & 12.1 \\
\hline 32 & BM 58 wk & $37,418,398$ & 168,215 & 64.5 & 64.6 & 23.6 & 11.9 \\
\hline
\end{tabular}


To characterize DNA methylation patterns, we employed Canine DREAM for all of 32 samples. From all 32 samples, 24.5-50.5 million unique usable sequencing reads after conservative filtering (quality filtered and aligned to the dog genome) were successfully generated for DNA methylation analyses (Table 1). We used CpG sites that had more than 20 reads (157-181 thousands CpG sites per sample) to assure quantitative ability. For all the analyses, only autosomal CpGs were considered, resulting in 130,861 common CpG sites obtained for inter-sample comparisons.

Of 130,861 CpG sites, 6416 (4.9\%) sites were located closely at a promoter region of genes annotated by Ensembl Gene Predictions - version 99.9002 (6.9\%) and 55,065 (42\%) sites were located at exons and introns of the genes, respectively. The remaining $60,378 \mathrm{CpG}$ sites were distant from any of the above gene annotations except for 2388 sites located on non-coding RNA such as IncRNA (Fig. 1a). CGIs have been recognized to be one of the most important methylation features of the genome and methylated differently from non-CpG island (NCGI) regions in mammals [42]. In this regard, 44,023 sites were in CGIs and 86,838 sites in NCGIs (Fig. 1b).

\section{Overall status of DNA methylation in each tissue}

Next, we addressed overall status of DNA methylation level in all tissue types analyzed. The average DNA methylation levels for each tissue type were $59.0 \%-65.1 \%$ with small levels of variation among tissue types. Figure $2 a$ and $2 b$ show DNA methylation fractions and the overall distribution of the DNA methylation levels. We found that the majority of $\mathrm{CpG}$ sites were either highly methylated (52.5\%-64.6\% of all CpG sites analyzed) or unmethylated (22.5\%-28.0\% of all CpG sites analyzed), and the remaining $11.9 \%-21.8 \%$ were intermediately methylated (Table 1).

Low level DNA methylation is thought to be a signature of gene regulation ${ }^{19,20}$. Therefore, we sought for the potential of each $\mathrm{CpG}$ site in the role in gene expression regulation by identifying the lowest DNA methylation level. We found that $53,830 \mathrm{CpG}$ sites (41.1\% of CpG sites analyzed) had their lowest DNA methylation level below $30 \%$. Even though roughly $25 \%$ of $\mathrm{CpG}$ sites were unmethylated (calculated by the average of the percentage of unmethylated $\mathrm{CpG}$ sites in all cell types) for each cell type, $41.1 \%$ of the $\mathrm{CpG}$ sites could be unmethylated to have potential regulatory functions.

We calculated the average methylation levels for different genomic features in all cell types and found relatively lower methylation levels in promoter regions compared to exons, introns, or intergenic regions. (Fig. 2c).

\section{Tissue specificity determined by genome-wide DNA methylation patterns}

To address if DNA methylome derived from different tissue types could identify tissue specificity, we calculated correlation coefficient for all the pairs (496 pairs) with samples analyzed. As expected, relatively high correlation $(R=0.84-0.99)$ were observed in all the pairs (Fig. 3a). However, the tissue types were clearly defined by Principal component analysis (Fig. 3b) and hierarchical clustering analysis (Fig. 3c). Interestingly, different gastrointestinal cell type methylomes (duodenum, stomach, ileum, and colon) and epithelial cell type methylomes (oral mucosa and skin) had notable similarity to each other.

\section{Classification of CpG sites by DNA methylation patterns across all samples}

The overall distributions of methylated or unmethylated $\mathrm{CpG}$ sites from the DNA methylation data of 32 samples were similar, however, genome-wide DNA methylation patterns were variable enough to identify difference of tissue types, indicating that methylation level could be either stable or variable across the samples. To address this hypothesis, we classified $\mathrm{CpG}$ sites into constitutively methylated (M), unmethylated (U), and intermediately methylated (I) using 70\% and $30 \%$ as cutoffs. This resulted in $48.7 \%$ of $\mathrm{CpG}$ sites being constitutively methylated (29.9\%) or unmethylated (18.9\%). Constitutively intermediately methylated $\mathrm{CpG}$ sites were found only in $1.1 \%$ of all $\mathrm{CpG}$ sites analyzed.

The majority (73.4\%) of constitutively unmethylated CpG sites were in CGIs and $41 \%$ of all the CpG sites in CGIs were constitutively unmethylated (Fig. 4a) whereas the majority of constitutively methylated CpG sites (76.2\%) and intermediately methylated CpG sites (80.2\%) were in NCGIs and $34.3 \%$ of all the CpG sites in NCGIs were constitutively methylated CpGs (Fig. 4a). These findings agreed well with generally appreciated DNA methylation patterns regarding CGI 21.

Notably, of 24,674 CpG constitutively unmethylated CpG sites, 4777 (19.4\%) sites were located closely at promoter region of genes, which was four times higher frequency than that of all CpG sites analyzed. 3898 (15.8\%) and 8387 (34.0\%) sites were located at exon and intron of genes, respectively (Fig. 4b).

Next, we sought for variable (V) CpG sites across the samples by utilizing definition of having a gap of at least $40 \%$ between the third highest and the third lowest methylation values. As a result, 22,385 (17.1\%) CpG sites were identified to be variable in our dataset (Fig. 4c), of which a substantial number of these sites located at intron ( $9981 \mathrm{CpG}$ sites) or intergenic regions (10,521 CpG sites), leaving only 449 (2.0\%) CpG sites located at promoter regions.

\section{DNA methylation level correlated with gene expression}

DNA methylation at promoter is known to correlates with gene expression silencing ${ }^{3}$. Of $130,861 \mathrm{CpG}$ sites, 6416 (4.9\%) sites were located closely at promoter region of genes, which corresponded to 7517 transcripts. We obtained RNA-seq data of the same samples and integrated available gene expression data to observe correlation between differential gene expression and DNA methylation. As a result, we could utilize $3783 \mathrm{CpG}$ sites that were located at promoter region of these transcripts. We plotted differential expression and methylation levels between each pair of all samples after averaging anatomically similar tissues (stomach, duodenum, ileum, and colon were combined intro Gl tract. Likewise, skin and oral mucosa samples were combined into Epithelial) (Fig. 5). All of 90 scatterplots showed negative correlation between the two parameters; hypermethylation with gene expression decrease and hypomethylation with gene expression increase between assigned different tissues $(r=-0.12--0.02$, Pearson's correlation).

Following differentially methylated regions identified in human, the differential methylation has been linked to tissue specific gene expression (Zhang et al. 2013; Lowdon et al. 2014). Therefore, we hypothesized uniquely methylated or unmethylated CpG sites were hallmarks of gene expression in different cell 
types. In this regard, we extracted $\mathrm{CpG}$ sites that were uniquely unmethylated in only one out of 11 tissue types for the following analysis. After the tissues were ranked by their DNA methylation level for individual CpG sites, we defined uniquely unmethylated sites as being only one tissue showing < $30 \%$ methylation with a gap of at least $30 \%$ between the lowest and the second lowest methylation values. As a result, we identified 1690 uniquely unmethylated sites and found 94 transcripts with these sites at promoter regions. To analyze the relationship between DNA methylation and gene expression for different tissue types, we utilized RNA-seq data for the same samples and found representative 10 transcripts with significantly lower gene expression in the tissue with the second highest DNA methylation levels (Table 2). One of the significantly correlated genes, PKP3, was known to play a role in cellular desmosomedependent adhesion and was found to be highly expressed in epithelial tissue as assumed from the function of the gene.

Table 2

Representative uniquely methylated $\mathrm{CpG}$ sites with genes

\begin{tabular}{|c|c|c|c|c|c|c|c|c|c|}
\hline $\begin{array}{l}\text { Chromosome } \\
\text { and position }\end{array}$ & ENSCAFT ID & $\begin{array}{l}\text { Gene } \\
\text { name }\end{array}$ & $\begin{array}{l}\text { Uniquely } \\
\text { methylated } \\
\text { tissue }\end{array}$ & $\begin{array}{l}\text { Methylation } \\
\text { in uniquely } \\
\text { methylated } \\
\text { tissue }\end{array}$ & $\begin{array}{l}\text { Expression } \\
\text { in uniquely } \\
\text { methylated } \\
\text { tissue }\end{array}$ & $\begin{array}{l}\text { Second } \\
\text { tissue }\end{array}$ & $\begin{array}{l}\text { Methylation } \\
\text { in Second } \\
\text { tissue }\end{array}$ & $\begin{array}{l}\text { Expression } \\
\text { in Second } \\
\text { tissue }\end{array}$ & $\begin{array}{l}\text { Expres } \\
\text { differe } \\
\text { (fold } \\
\text { chang }\end{array}$ \\
\hline chr18:25539224 & ENSCAFT00000010495.3 & PKP3 & Epithelial & 26.1 & 316.2 & Muscle & 78.2 & 0.5 & 632.4 \\
\hline chr7:43493723 & ENSCAFT00000027812.4 & S100A5 & Epithelial & 27.7 & 286.9 & GI tract & 74.4 & 64.2 & 4.5 \\
\hline chr7:1610651 & ENSCAFT00000044289.3 & TNNT2 & Heart & 22.1 & 1737.2 & Epithelial & 69.2 & 3.6 & 488.5 \\
\hline chr7:1610651 & ENSCAFT00000043795.3 & TNNT2 & Heart & 22.1 & 20.9 & Epithelial & 69.2 & 2.8 & 7.3 \\
\hline chr20:37051632 & ENSCAFT00000081514.1 & $\mathrm{ITIH} 1$ & Liver & 22.8 & 731.4 & Pancreas & 80.0 & 0.5 & 1462.i \\
\hline chr8:68602234 & ENSCAFT00000028408.4 & SLC25A47 & Liver & 26.0 & 479.4 & Muscle & 72.8 & 0.5 & 958.8 \\
\hline chr10:1130973 & ENSCAFT00000000245.4 & RDH16 & Liver & 19.0 & 410.6 & Lung & 58.9 & 36.9 & 11.1 \\
\hline chr5:75513913 & ENSCAFT00000031965.4 & CTRB2 & Pancreas & 24.2 & 41237.5 & Spleen & 86.2 & 15.4 & 2679.; \\
\hline chr3:91435257 & ENSCAFT00000026438.2 & TMED11 & Pancreas & 15.4 & 138.4 & Kidney & 77.0 & 0.5 & 276.8 \\
\hline chr14:6569061 & ENSCAFT00000082881.1 & CPA1 & Pancreas & 19.4 & 15.0 & Muscle & 73.8 & 0.5 & 30.0 \\
\hline
\end{tabular}

\section{Discussion}

We previously reported Canine DREAM, which is a genome-wide DNA methylation analysis of the dog genome ${ }^{18}$. We utilized Canine DREAM in this study for a variety of normal tissues to construct DNA methylation profiles in dogs and provide basic information for improving our understanding. We analyzed 130,861 CpG sites detected in all samples, which is much more than generally reported DNA methylation studies with strategy that targeted genes of interest, suggesting that Canine DREAM can provide a more in-depth view of DNA methylation status than single-locus studies ${ }^{16,22}$.

The average DNA methylation levels of all the $\mathrm{CpG}$ sites as well as overall distribution of the DNA methylation levels analyzed for each tissue type were quite similar with small levels of variation among tissue types. These findings in the dog were consistent with other species. In this study, the 16 dog tissues showed similar global methylation with correlation coefficients ranging from 0.84 to 0.99 . Previously, ten bovine tissues showed correlations ranging from 0.93 to $0.98^{23}$. The pig study with closely related tissues yielded slightly higher correlations $(>0.95)^{24}$.

Particularly, $29.9 \%$ of $\mathrm{CpG}$ sites were constitutively methylated regardless of the cell types whereas $18.9 \%$ of CpG sites were constitutively unmethylated. The fact that approximately half of CpG sites are stable in all the 32 samples analyzed in this study is consistent with the fact that DNA methylation is a stable mark across different dog cell types. The majority (73.4\%) of constitutively unmethylated CpGs were in CGI, and the majority of constitutively methylated CpG sites $(76.2 \%)$ and intermediately methylated CpG sites (80.2\%) were in NCGIs, which is consistent with the notion that the CGIs were generally less methylated than the NCGIs ${ }^{21}$ and also that methylated NCGIs are suggested to suppress unnecessary retrotransposon expression ${ }^{25}$.

Although overall DNA methylation status among tissue types was similar, principal component analysis and hierarchical clustering analysis with all 32 methylome data showed clear differences indicating tissue-specific DNA methylation patterns. This is consistent with the reports of human and bovine methylome where cell/tissue types were also separated clearly by similar analyses ${ }^{23,26}$. These results suggest that DNA methylation patterns are profoundly involved in tissue differentiation across species. In any given cell type, approximately the same percentage of CpG sites were found methylated and unmethylated, however, methylation status of $\mathrm{CpG}$ sites in a certain cell type could be cell-type dependent.

Since our findings indicated different DNA methylome depending on tissue types, we sought for tissue-specific DNA methylation that were associated with gene expression regulation. DNA methylation in promoter region of genes is associated with gene silencing ${ }^{27}$. This was supported by our results that DNA methylation in the promoter regions showed largely negative correlation with gene expression. In addition, we filtered uniquely unmethylated $\mathrm{CpG}$ sites at promoter regions in only one tissue type to identify exclusive expression characteristics. As a result, 0 (Adrenal gland) - 434 (Pancreas) uniquely methylated CpG sites were found and this variable numbers of uniquely unmethylated $\mathrm{CpG}$ sites could partly be attributed to the tissue characteristics in this study.

Widespread colocalization between transcription factor binding and variably methylated $\mathrm{CpG}$ sites outside promoter regions were reported in humans ${ }^{20,26}$. Although 22,385 variably methylated CpG sites were found in this study, the majority ( $>91 \%)$ of these sites located at intron or intergenic regions and could not be analyzed systematically in this regard due to lack of these data in the dog genome. It is no wonder that those variably methylated $\mathrm{CpG}$ sites that did 
overlap with nothing on currently available database might harbor roles in regulation of transcription factor binding which would be identified by increasing amount of transcription factor and histone modification ChIP-seq data in the future.

We expect that more variably and uniquely methylated $\mathrm{CpG}$ sites will be identified if more cell types are sequenced and analyzed in the future. We would clearly suggest that DNA methylation plays a role in the regulation of cell or tissue type-specific gene functions. The data and results provided in this study will be useful in the research field of veterinary medicine as well as human medicine. Our methylation data would also be helpful in interpreting the epigenomic status in a variety of cells and conditions such as tumors. It is important to remind that Canine DREAM only analyzed a small part of the dog genome, and more extensive studies such as whole genome bisulfite sequencing are needed to confirm these findings. Nevertheless, our data and many more single-CpGresolution DNA methylome data available in the future will provide greater insights into the knowledge of epigenetics research field.

\section{Methods}

\section{Dogs}

Two female Beagles 58-week and 73-week-old were used as healthy controls. These dogs were healthy, had no clinical signs, no abnormalities in urinalysis, hematological examination or blood biochemical analysis. No parasites or pathogenic bacteria were detected in fecal samples. The use of dogs in this study was approved by the Animal Care Committee of the University of Tokyo (Approval No. P17-064). Food was withheld from each dog for 12 hours. Dogs then were euthanized and 16 tissues including, lung, heart, stomach, duodenum, ileum, colon, liver, pancreas, adrenal gland, kidney, spleen, muscle, skin (white), oral (white), oral (black), and bone marrow were obtained. All specimens for Canine DREAM was stored at $-80^{\circ} \mathrm{C}$.

\section{Digital restriction enzyme analysis of methylation (DREAM)}

Genome-wide DNA methylation analysis using next-generation sequencing was performed as previously ${ }^{18}$ with genomic DNA ( $\left.2 \mu \mathrm{g}\right)$ extracted from the above samples. We used the University of California, Santa Cruz (UCSC) definition of CpG islands ${ }^{28}$. Promoter regions are defined as being located within $1 \mathrm{~kb}$ from transcription start sites of given genes.

\section{RNA-Seq analysis}

Total RNA was extracted by the NucleoSpin RNA (Macherey-Nagel, Duren, Germany) for each tissue. RNA integrity number of all RNA is confirmed to be more than 7.0. The RNA-Seq library was constructed by SMART-Seq v4 (Takara Bio) and Nextera XT DNA Library Kit (Illumina). Sequencing was performed with Illumina NovaSeq 6000 according to the manufacture's software. Quality filtering for sequence reads were performed using Trim Galore (version 0.6.4) with the Phred cutoff score of 30. We used 32-42 million reads after quality control of sequenced reads. Transcripts per million (TPM) was calculated to evaluate gene expression by using Kalisto (version 0.46.2) with default settings and dog gene annotation data sets (CanFam3.1).

\section{Data analysis and visualization}

Violin plots, density plots, boxplots, and Starburst plots were drawn and visualized with Seaborn on Python 3.7. Hierarchical clustering analysis was performed with the agglomeration method 'ward' where the distance was calculated with the Euclidean and visualized with Seaborn on Python 3.7. Principal component analysis was performed using $\mathrm{R}$ with a package ggfortify.

\section{Abbreviations}

CGI: CpG islands

DREAM: Digital Restriction Enzyme of DNA Methylation Analysis

\section{Declarations}

\section{Data availability}

The dataset generated and/or analysed during the current study are underway to be submitted to Gene Expression Omnibus (GEO) and will be available once it is accepted.

\section{CONFLICT OF INTEREST DECLARATION}

Authors declare no conflicts of interest.

\section{INSTITUTIONAL ANIMAL CARE AND USE COMMITTEE (IACUC) OR OTHER APPROVAL DECLARATION}

This study was approved by the Animal ethical committee of The University of Tokyo.

\section{HUMAN ETHICS APPROVAL DECLARATION}

Authors declare human ethics approval was not needed for this study. 


\section{Author contributions}

J.Y. designed the study. S.M. provided resources. J.Y., Y.M., T.I. and K.W. conducted the experiment(s). J.Y., Y.M., J.J. and J.Y. analysed the data. J.Y., Y.M. and J.J. wrote and edited the manuscript. G.I. and M.T. supervised project administration. All authors reviewed the manuscript.

\section{Corresponding author}

Correspondence to Jumpei Yamazaki

\section{References}

1. Razin, A. \& Cedar, H. DNA methylation and gene expression. Microbiol. Rev. 55, 451-458 (1991).

2. Illingworth, R. S. \& Bird, A. P. CpG islands-'a rough guide'. FEBS Lett. 583, 1713-1720 https://doi.org/10.1016/j.febslet.2009.04.012 (2009).

3. Jones, P. A. \& Baylin, S. B. The epigenomics of cancer. Cell. 128, 683-692 https://doi.org/10.1016/j.cell.2007.01.029 (2007).

4. Heard, E., Clerc, P. \& Avner, P. X-chromosome inactivation in mammals. Annu. Rev. Genet. 31, 571-610 https://doi.org/10.1146/annurev.genet.31.1.571 (1997).

5. Barlow, D. P. Gametic imprinting in mammals. Science. 270, 1610-1613 (1995).

6. Ferguson-Smith, A. C. Genomic imprinting: the emergence of an epigenetic paradigm. Nat Rev Genet. 12, 565-575 https://doi.org/10.1038/nrg3032 (2011).

7. Figueroa, M. E. et al. MDS and secondary AML display unique patterns and abundance of aberrant DNA methylation. Blood. 114, 3448-3458 https://doi.org/10.1182/blood-2009-01-200519 (2009).

8. Noushmehr, H. et al. Identification of a CpG island methylator phenotype that defines a distinct subgroup of glioma. Cancer Cell. 17, 510-522 https://doi.org/10.1016/j.ccr.2010.03.017 (2010).

9. Lister, R. et al. Human DNA methylomes at base resolution show widespread epigenomic differences. Nature. 462, 315-322 https://doi.org/10.1038/nature08514 (2009).

10. Schultz, M. D. et al. Human body epigenome maps reveal noncanonical DNA methylation variation. Nature. 523, 212-216 https://doi.org/10.1038/nature14465 (2015).

11. Habibi, E. et al. Whole-genome bisulfite sequencing of two distinct interconvertible DNA methylomes of mouse embryonic stem cells. Cell Stem Cell. 13, 360-369 https://doi.org/10.1016/j.stem.2013.06.002 (2013).

12. Kirkness, E. F. et al. The dog genome: survey sequencing and comparative analysis. Science. 301, 1898-1903 https://doi.org/10.1126/science.1086432 (2003).

13. Paoloni, M. \& Khanna, C. Translation of new cancer treatments from pet dogs to humans. Nat. Rev. Cancer. 8, 147-156 https://doi.org/10.1038/nrc2273 (2008).

14. Morimoto, C. Y. et al. Evaluation of the global DNA methylation in canine mast cell tumour samples by immunostaining of 5-methyl cytosine. Vet. Comp. Oncol. https://doi.org/10.1111/vco.12241 (2016).

15. Yamaya, Y., Sugiya, H. \& Watari, T. Methylation of free-floating deoxyribonucleic acid fragments in the bronchoalveolar lavage fluid of dogs with chronic bronchitis exposed to environmental tobacco smoke. Ir. Vet. J. 68, 7 https://doi.org/10.1186/s13620-015-0035-4 (2015).

16. Ferraresso, S. et al. Epigenetic silencing of TFPI-2 in canine diffuse large B-cell lymphoma. PLoS One. 9, e92707 https://doi.org/10.1371/journal.pone.0092707 (2014).

17. Tomiyasu, H. et al. Evaluation of DNA methylation profiles of the CpG island of the ABCB1 gene in dogs with lymphoma. Am. J. Vet. Res. 75, 835-841 https://doi.org/10.2460/ajvr.75.9.835 (2014).

18. Yamazaki, J., Jelinek, J., Hisamoto, S., Tsukamoto, A. \& Inaba, M. Dynamic changes in DNA methylation patterns in canine lymphoma cell lines demonstrated by genome-wide quantitative DNA methylation analysis. Vet. J. 231, 48-54 https://doi.org/10.1016/j.tvjl.2017.11.007 (2018).

19. Tsankov, A. M. et al. Transcription factor binding dynamics during human ES cell differentiation. Nature. 518, 344-349 https://doi.org/10.1038/nature14233 (2015).

20. Stadler, M. B. et al. DNA-binding factors shape the mouse methylome at distal regulatory regions. Nature. 480, 490-495 https://doi.org/10.1038/nature10716 (2011).

21. Bird, A. DNA methylation patterns and epigenetic memory. Genes Dev. 16, 6-21 https://doi.org/10.1101/gad.947102 (2002).

22. Bryan, J. N. et al. Hypermethylation of the DLC1 CpG island does not alter gene expression in canine lymphoma. BMC Genet. 10,73 https://doi.org/10.1186/1471-2156-10-73 (2009).

23. Zhou, Y. et al. Reduced representation bisulphite sequencing of ten bovine somatic tissues reveals DNA methylation patterns and their impacts on gene expression. BMC Genomics. 17, 779 https://doi.org/10.1186/s12864-016-3116-1 (2016).

24. Choi, M. et al. Genome-wide analysis of DNA methylation in pigs using reduced representation bisulfite sequencing. DNA Res. 22, 343-355 https://doi.org/10.1093/dnares/dsv017 (2015).

25. Zhou, W., Liang, G., Molloy, P. L. \& Jones, P. A. DNA methylation enables transposable element-driven genome expansion. Proc. Natl. Acad. Sci. U. S. A. 117, 19359-19366, doi:10.1073/pnas.1921719117 (2020). 
26. Gu, J. et al. Mapping of Variable DNA Methylation Across Multiple Cell Types Defines a Dynamic Regulatory Landscape of the Human Genome. G3 (Bethesda). 6, 973-986 https://doi.org/10.1534/g3.115.025437 (2016).

27. Varley, K. E. et al. Dynamic DNA methylation across diverse human cell lines and tissues. Genome Res. 23, 555-567 https://doi.org/10.1101/gr.147942.112 (2013).

28. Gardiner-Garden, M. \& Frommer, M. CpG islands in vertebrate genomes. J. Mol. Biol. 196, 261-282 (1987).

\section{Figures}

\section{Fig.1}

a

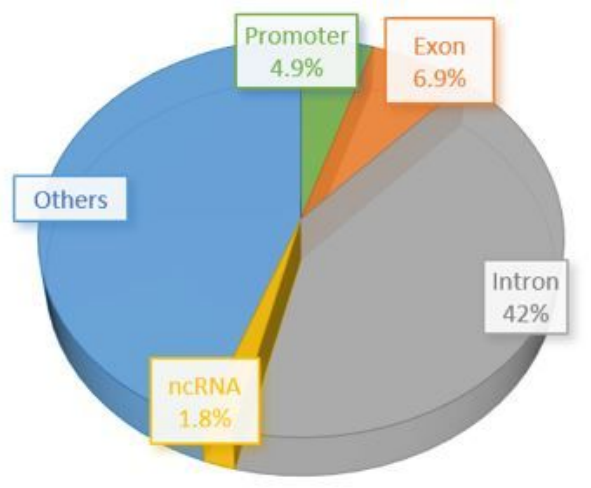

b

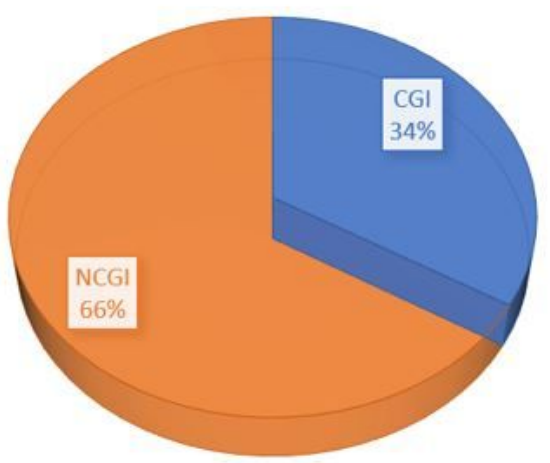

\section{Figure 1}

Percentage of $\mathrm{CpG}$ sites analyzed in this study in each category of (a) genomic features and (b) CpG islands. 
Fig. 2

a

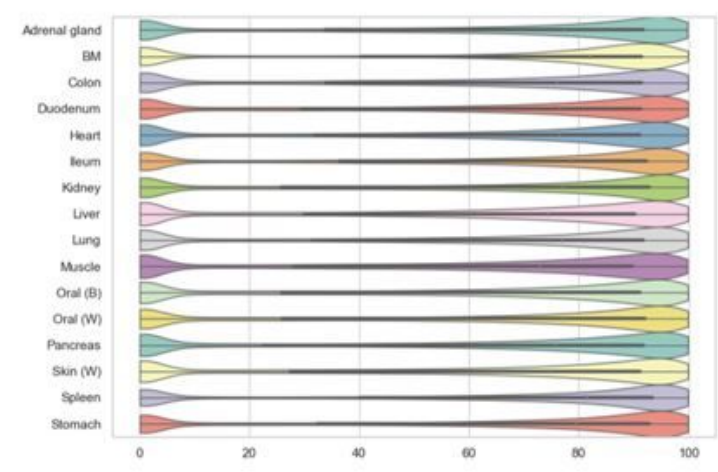

C

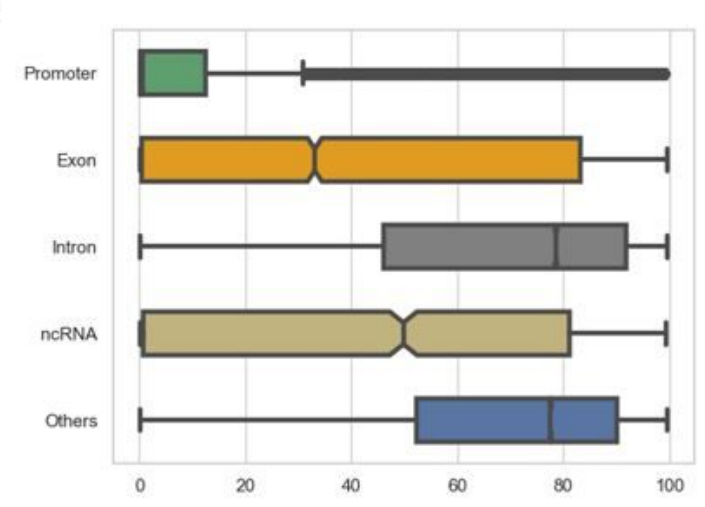

\section{b}

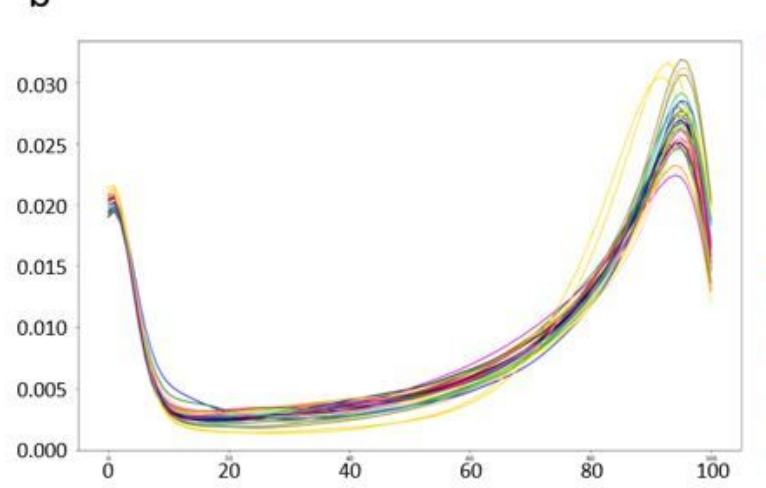

- tung 73

- stomach 73 — Dudenum 13 - lleum 73 - Colonn 7

- Liver 73

- Pancreas 73

- idrenal gland 73
Kothey 73

Kodney 73

- Spleen 73

- Musde 73

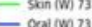

二 Oral (8) 73

- 8973

- lung 58

二 Hear 58

二 Stomach 58

- leum 58

- colon 58

- Liver 58

- Pancreas 58 .

Koney 58
- Selen 58

- Spleen 58

- Muscies

— Oral (WI) 58

- oral (8) 58

- cral (8) 58
- BM 58

\section{Figure 2}

Characterization of DNA methylation patterns across cell types (A) Violin plots of genome-wide DNA methylation levels of all CpG sites for each of the 16 methylome after averaging values for the same tissues. (B) Density plots of genome-wide DNA methylation levels of all CpG sites for each of the 32 methylomes. (C) DNA methylation level of all $\mathrm{CpG}$ sites classified by the genomic feature. 
Fig.3

a

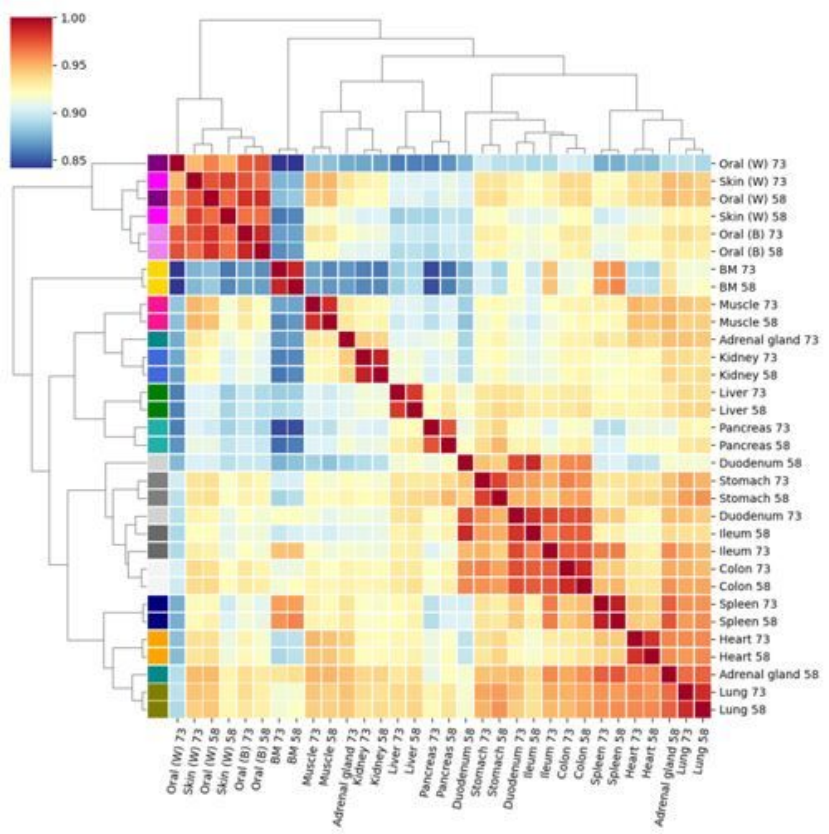

b

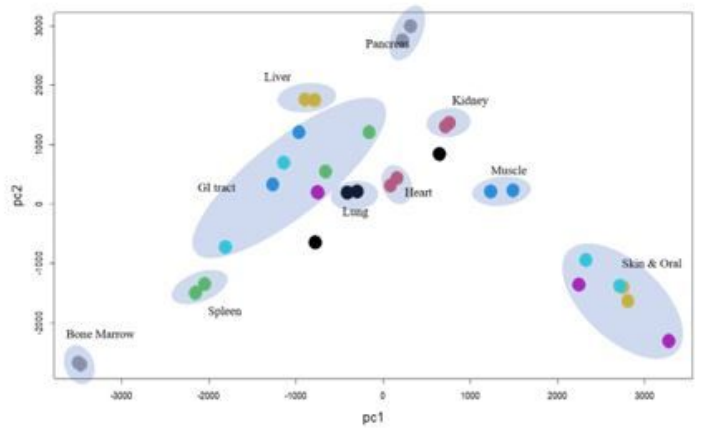

C

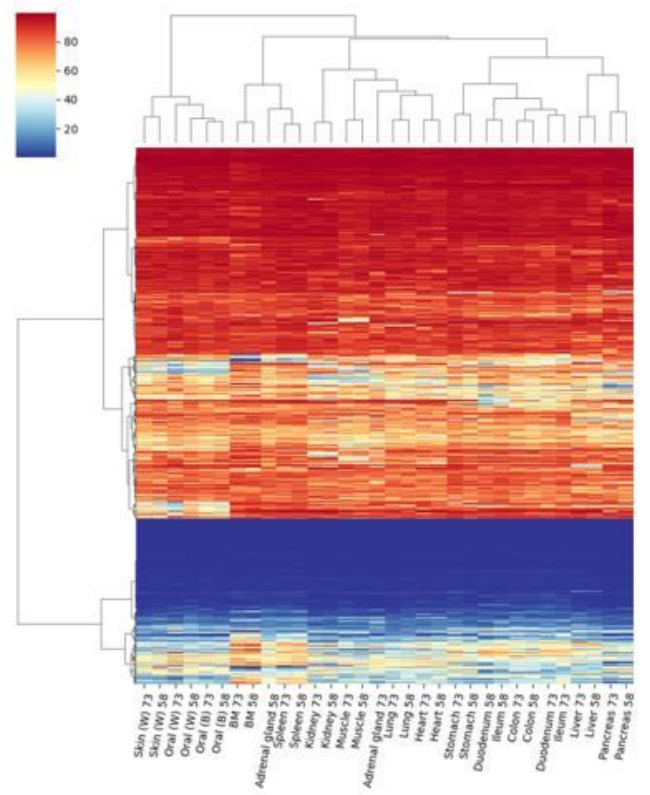

\section{Figure 3}

(A) Pairplot for correlation scores of DNA methylation levels with all CpG sites analyzed by Canine DREAM. Samples include lung, heart, stomach, duodenum, ileum, colon, liver, pancreas, adrenal gland, kidney, spleen, muscle, skin (white-colored), oral mucosa (white-colored and black-colored), and bone marrow. Also shown is (B) Principal Component Analysis and (C) Unsupervised hierarchical analyses of DNA methylation levels with all CpG sites analyzed by Canine DREAM. 
Fig.4

a
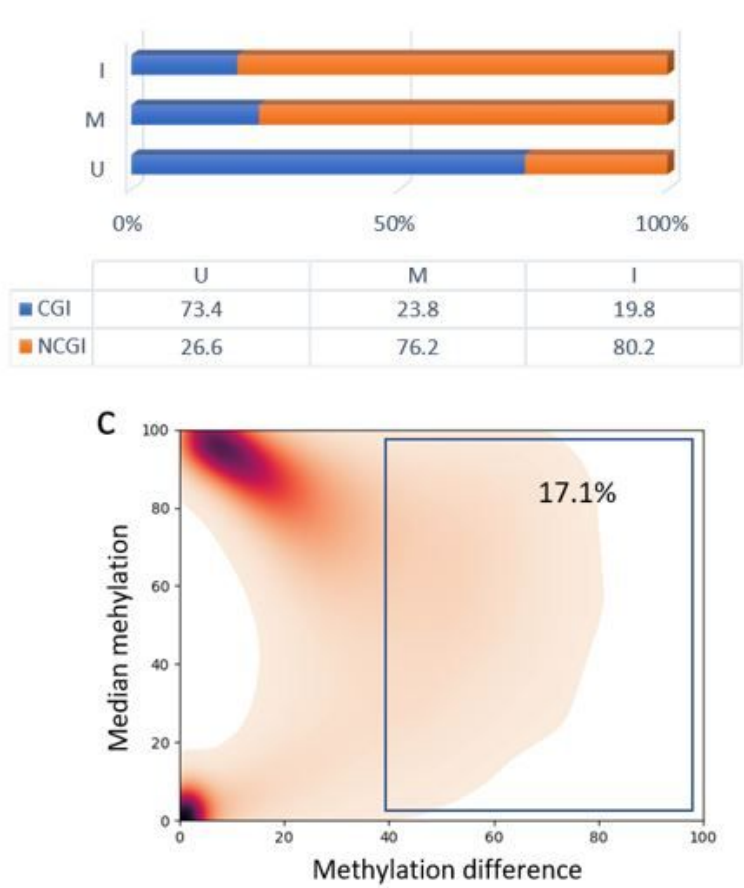

b

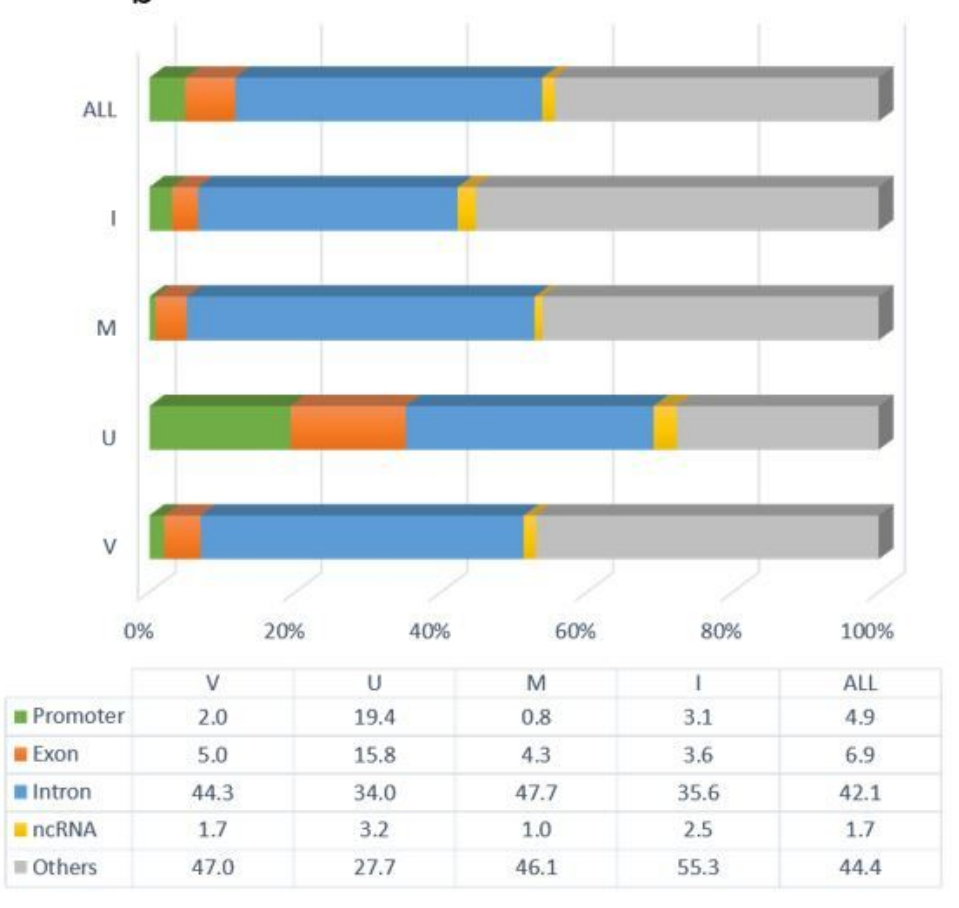

\section{Figure 4}

Percentage of constitutively unmethylated (U), methylated (M), and intermediately methylated (I) CpGs analyzed in this study in each category of (a) CpG islands and (b) genomic features. (c) Density scatterplot of CpG-wise DNA methylation level differences (x axis) and CpG median methylation (y axis) across the 32 samples. Coloring indicates $\mathrm{CpG}$ density from low (sparse) to high (dense). The blue box highlights variable $\mathrm{CpG}$ sites. 
Fig.5

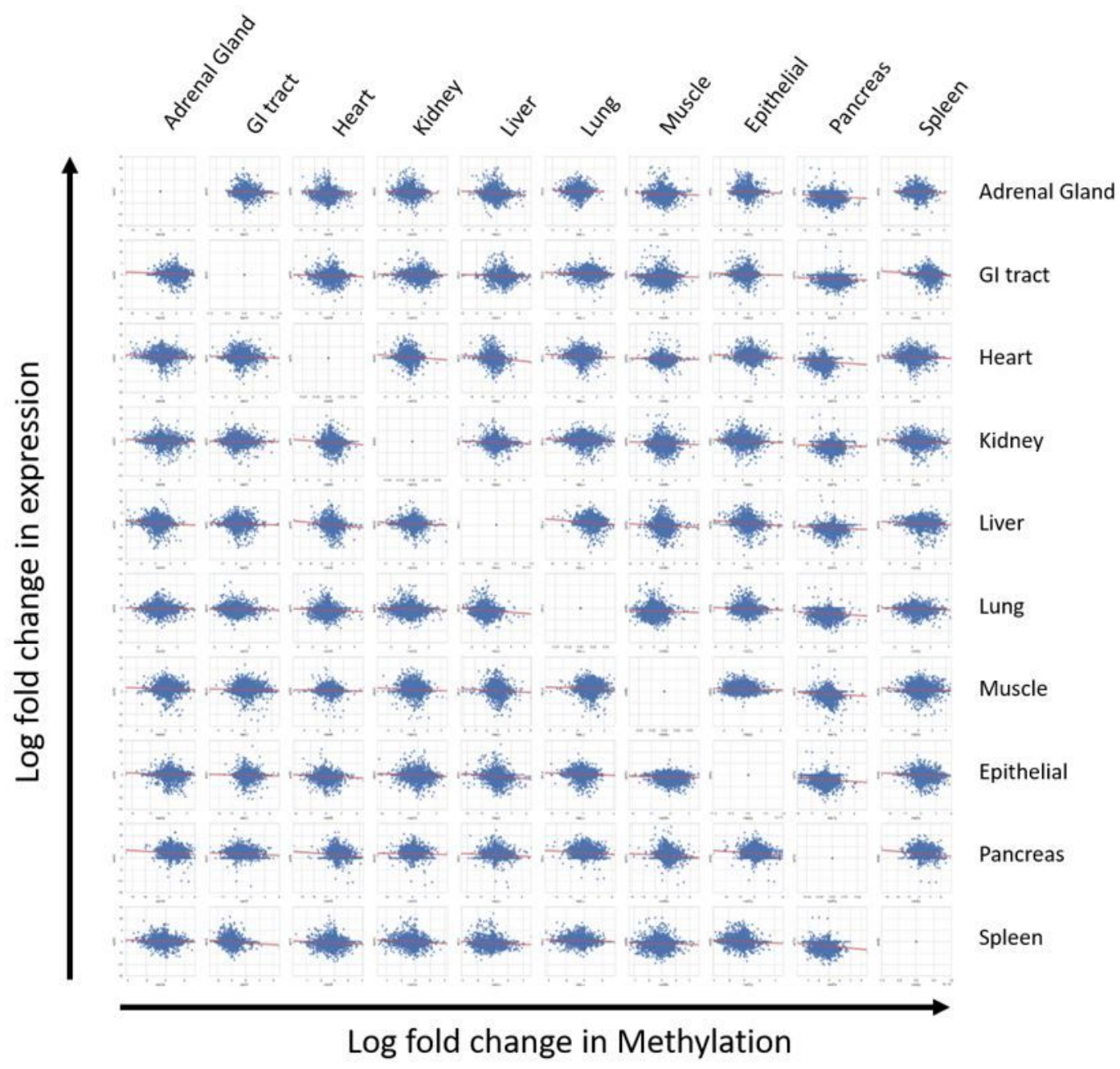

\section{Figure 5}

Integration of methylation and expression data. Starburst plot of the $3783 \mathrm{CpG}$ sites that were located at promoter region of transcripts whose gene expression data were available. Log fold change in DNA methylation ( $x$-axis) and gene expression ( $y$-axis) are plotted for every comparison between each tissue. When the RPKM for a transcript is less than 0.5 , the value was adjusted to 0.5 . Log fold change were calculated from the value of tissues shown in a row subtracted by tissues in a column. 